\title{
Set Theoretical Variants of Optimization Algorithms for System Reliability-based Design of Truss Structures
}

\author{
Ali Kaveh $^{1 *}$, Kiarash Biabani Hamedani ${ }^{1}$, Mohammad Kamalinejad ${ }^{1}$ \\ ${ }^{1}$ School of Civil Engineering, Iran University of Science and Technology, Narmak, 16846-13114, Tehran, Iran \\ * Corresponding author, e-mail: alikaveh@iust.ac.ir
}

Received: 15 November 2020, Accepted: 21 January 2021, Published online: 05 February 2021

\begin{abstract}
In this paper, recently developed set theoretical variants of the teaching-learning-based optimization (TLBO) algorithm and the shuffled shepherd optimization algorithm (SSOA) are employed for system reliability-based design optimization (SRBDO) of truss structures. The set theoretical variants are designed based on a simple framework in which the population of candidate solutions is divided into some number of smaller well-arranged sub-populations. In addition, the framework is applied to the Jaya algorithm, leading to a set-theoretical variant of the Jaya algorithm. So far, most of the reliability-based design optimization studies have focused on the reliability of single structural members. This is due to the fact that the optimization problems with system reliability-based constraints are computationally expensive to solve. This is especially the case of statically redundant structures, where the number of failure modes is so high that it is impractical to identify all of them. System-level reliability analysis of truss structures is carried out by the branch and bound method by which the stochastically dominant failure paths are identified within a reasonable time. At last, three numerical examples, including size optimization of truss structures, are presented to illustrate the effectiveness of the proposed SRBDO approach. The results indicate the efficiency and applicability of the set theoretical optimization algorithms to solve the SRBDO problems of truss structures.
\end{abstract}

\section{Keywords}

teaching-learning-based optimization algorithm, shuffled shepherd optimization algorithm, Jaya algorithm, truss structure, structural optimization, structural reliability theory

\section{Introduction}

Truss optimization is one of the most interesting topics in structural optimization. Optimization of truss structures can be classified into three categories of size optimization, shape optimization, and topology optimization [1]. Moreover, two or all three of the aforementioned categories can be considered simultaneously. The general purpose for truss optimization problems is to obtain a truss structure with minimum weight that satisfies a set of predefined constraints. In the last three decades, the application of metaheuristic algorithms to deterministic design optimization of truss structures has attracted the interest of many researchers. For example, Dhingra and Bennage [2] applied simulated annealing (SA) algorithm for topological optimization of truss structures. Kaveh and Khayatazad [3] applied ray optimization (RO) algorithm to solve truss optimization problems. Hasançebi and Kazemzadeh Azad used a refined version of the big bang-big crunch (BB-BC) algorithm [4] and the adaptive dimensional search (ADS) algorithm [5] for discrete truss sizing optimization problems. Recently, Kaveh et al. [6, 7] applied set theoretical variants of some of population-based metaheuristic algorithms to solve frequency-constrained truss optimization problems. Nonetheless, most of the published researches are limited to deterministic design approaches where partial safety factors are used to design sufficiently safe structures. Although deterministic design approaches provide appropriate degrees of structural reliability, they cannot model uncertainties of the structure. However, it has been recognized for many decades that material properties, loads, and geometrical dimensions are in general associated with some uncertainties. Hence, deterministic design approaches cannot provide means to assess the reliability and safety of the structures, which may result to overestimated designs in many cases. On the 
other hand, reliability-based design approaches make it possible to directly take into account the aforementioned uncertainties in the design process. As a consequence, in recent years, several studies have been focused on the reliability-based design optimization (RBDO) approaches in which the structural failure probability is directly considered as the optimization constraint [8-10]. For example, Nakib and Frangopol [11] performed reliability-based optimization of structures using a nonlinear mathematical programming package. Murotsu and Shao [12] presented an approach to the optimum shape design of truss structures based on reliability constraints. Murotsu et al. [13] proposed an optimum design procedure for redundant structures based on the constraints on formation probabilities of failure modes. Nakib [14] reported analytical investigation into both deterministic and reliability-based optimization of truss bridges. Stocki et al. [15] studied on discrete optimization techniques in reliability-based optimization of truss structures. Burton and Hajela [16] investigated the efficiency of a number of reliability-based optimization techniques in the shape optimization of truss structures. Dimou and Koumousis [17] employed the particle swarm optimization method for the reliability-based optimal design of statically determinate truss structures. Liu et al. [18] proposed a new hybrid method for system reliability-based optimization of truss structures based on genetic algorithm and neural network. Lopez et al. [19] employed a firefly algorithm and presented an approach for reliability-based design optimization of truss structures. Cheng and Jin [20] developed a reliability-based optimization approach and applied it to minimize the weight of steel truss arch bridges subject to deterministic and probabilistic constraints. Truong and Kim [21] used an improved differential evolution algorithm for sizing reliability-based design optimization of nonlinear inelastic truss structures. Palizzolo and Tabbuso [22] presented a framework for the RBDO of dynamically loaded elastic perfectly plastic truss structures. Zaeimi and Ghoddosian [23] performed system reliability based design optimization of truss structures with interval variables. Some recent RBDO studies have focused on the uncertainties related to the loading direction and magnitude [24-26].

It should be pointed out that achieving a sufficiently satisfactory estimate of the reliability of real structural systems requires a system approach [27]. In general, the approaches for reliability analysis of complex structural systems can be classified into three main categories: (a) numerical integration-based methods, (b) simulation-based methods, and (c) failure path-based methods [28]. System-level reliability analysis of structures is a time-consuming and complicated process, especially for the case of real redundant structures. Consequently, most of the RBDO problems have been solved based on the reliability of single structural members [15-16]. Only some simple structures have been solved based on system reliability constraints [12, 27].

In order to provide a practical tool for reliability-based design of truss structures, this research presents a SRBDO approach by employing: (1) a set theoretical framework for population-based optimization algorithms proposed by Kaveh et al. [6, 7]; and (2) the branch and bound method for system-level reliability analysis of truss structures. The loads and the strengths of structural members are considered to be normally distributed uncorrelated random variables. The structural members can be considered perfectly ductile or perfectly brittle. Moreover, buckling failure of the compression members is included. Some numerical examples of size optimization of truss structures are carried out to provide evidence to support the applicability and validity of the approach.

The rest of this paper is organized as follows: In Section 2 set theoretical variants of three population-based optimization algorithms are presented. Section 3 is devoted to system reliability analysis of truss structures. Section 4 presents formulation of system reliability-based design optimization problems for truss structures. In Section 5, the set theoretical variants of the optimization algorithms are utilized to solve some SRBDO problems of truss structures. Finally, the last section concludes the paper.

\section{Set theoretical variants of the population-based optimization algorithms}

Set theory is a branch of mathematics concerned with the study of properties of sets. A set is a well-defined collection of distinct objects. The objects of a set are called its elements or members. Any mathematical concept can be modelled by set theory. Thus, set theory can be employed as a foundation for mathematics. Set theory has found applications in structural engineering. For instance, Kaveh et al. [29] employed the set theory concepts to generalize the SSOA algorithm, and Kaveh et al. [6, 7] proposed a set theoretical framework for population-based metaheuristics.

Metaheuristics can be classified based on many different criteria, the most common of which is population-based search versus single-solution-based search. Populationbased metaheuristics (P-metaheuristics) require a set of candidate solutions to operate their own search process, 
while single-solution ones manipulate a single candidate solution during the search process. The initial population of P-metaheuristics can be viewed as a set with a certain number of elements. Recently, Kaveh et al. [6, 7] proposed a set theoretical framework for P-metaheuristics and developed set theoretical variants of two P-metaheuristics, the teaching-learning-based optimization algorithm and the shuffled shepherd optimization algorithm. The main idea of the framework is based on the division of the initial population into a number of smaller well-arranged sub-populations through which the search process continues. The framework aims to improve the compromise between exploration and exploitation of the search [6]. In the next two subsections, set theoretical variants of the teaching-learning-based optimization algorithm and shuffled shepherd optimization algorithm are presented briefly. Subsequently, set theoretical variant of the Jaya algorithm is developed.

\subsection{Set theoretical variants of the teaching-learning- based optimization algorithm}

The teaching-learning-based optimization (TLBO) algorithm is one of the most well-known P-metaheuristics developed by Rao et al. [30] in 2011. The TLBO algorithm is inspired by the traditional process of education in schools. A set or a class of learners is considered as the population of candidate solutions. The main body of the TLBO algorithm consists of two sequential phases of teacher and learner. The main idea of set theoretical variants of the TLBO algorithm is to divide the main class into a number of smaller well-arranged sub-classes of the same size. Kaveh et al. [6] developed two set theoretical variants of the TLBO algorithm, ordered set theoretical TLBO (OST-TLBO) and set theoretical multi-phase TLBO (STMP-TLBO). The set theoretical variants of the TLBO algorithm are presented as follows:

\subsubsection{Ordered set theoretical teaching-learning-based optimization (OST-TLBO) algorithm}

The OST-TLBO algorithm is stated in the following four steps [6]:

- Step one (initialization): The initial population is generated randomly.

- Step two (forming the sub-populations): The sub-populations are formed based on a procedure proposed by Kaveh et al. [29] as follows: Let us consider an initial population containing $n E$ candidate solutions. The aim is to form a certain number (e.g., $m$ ) of sub-populations of the same size. First, the population is sorted in ascending order of their penalized objective function values. Next, in the first step of forming the sub-populations, the first $m$ candidate solutions of the sorted population are selected and each element is placed randomly in one of the sub-populations. In the second step, the next $m$ candidate solutions are placed randomly in the sub-populations. The process continues until the entire candidate solutions are placed in the sub-populations. After completing the process, each sub-population contains $n S=n E / m$ candidate solutions.

- Step three (main body of TLBO): The main body of the TLBO algorithm is performed for all of the sub-populations. In other words, the teacher and learner phases are performed once for all of the sub-populations. After each phase, the replacement strategy is performed to keep the old solutions or replace them with the new ones.

- Step four (termination criteria): If the termination criterion of the algorithm is fulfilled, the algorithm terminates; otherwise, the algorithm returns to the second step.

Fig. 1 shows the steps of the OST-TLBO algorithm.

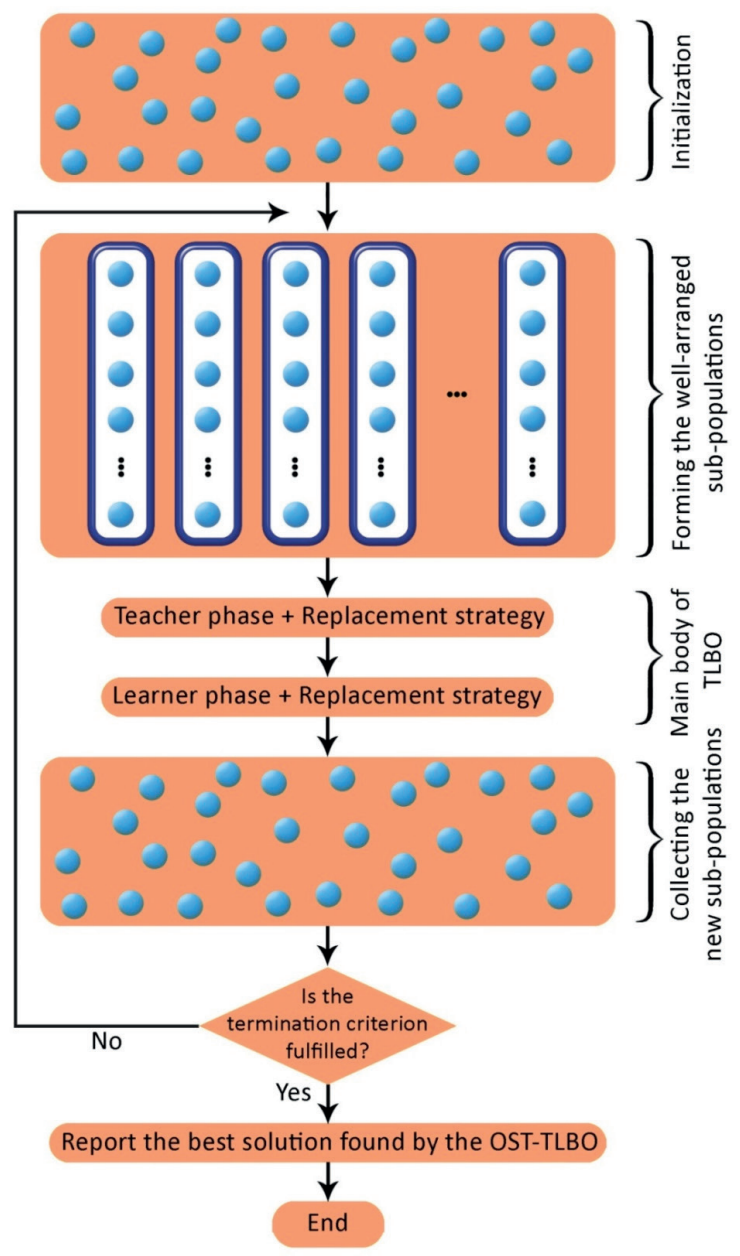

Fig. 1 Steps of the OST-TLBO algorithm 


\subsubsection{Set theoretical multi-phase teaching-learning- based optimization (STMP-TLBO) algorithm}

The STMP-TLBO algorithm is a multi-phase version of the OST-TLBO algorithm. Within each phase of the STMPTLBO algorithm, a self-contained OST-TLBO algorithm with a specific number of sub-populations is executed. The output of each phase is used as the input of its next phase. Each phase continues until the number of objective function evaluations (NFEs) reaches a predefined value. The only difference between the phases is the number of sub-populations. The number of sub-populations decreases from an integer number (e.g., $n_{0} ; 2<n_{0} \leq n E$ ) at the first phase to two at the last (e.g., $k$-th) phase. The steps of the STMP-TLBO algorithm are shown in Fig. 2. For more details, please refer to [6].

\subsection{Set theoretical variant of the shuffled shepherd optimization algorithm}

The shuffled shepherd optimization algorithm (SSOA) is a P-metaheuristic which mimics the herding behaviour of shepherds. Kaveh et al. [29] generalized the SSOA algorithm employing the concepts of set theory. Kaveh et al. [7] proposed two enhanced versions of the SSOA algorithm, parameter-free shuffled shepherd optimization algorithm (PF-SSOA) and set theoretical multi-phase shuffled shepherd optimization algorithm (STMP-SSOA). The STMPSSOA is a multi-phase variant of PF-SSOA. In each phase of the STMP-SSOA, a self-contained PF-SSOA with a specific number of sub-populations is performed. In other words, the phases of the STMP-SSOA differ in the number of sub-populations. As the optimization process continues, the number of sub-populations decreases. Each phase continues until the number of objective function evaluations (NFEs) reaches a predefined value. For more details, please refer to [7]. The steps of the STMP-SSOA are shown in Fig. 3. The PF-SSOA is stated in the following four steps [7]:

- Step one (initialization): The initial population is generated randomly.

- Step two (forming the sub-populations): The sub-populations are formed based on the procedure described in the second step of the OST-TLBO algorithm.

- Step three (solutions movement): The new position of the $j$-the candidate solution of the $i$-th sub-population $\left(\right.$ newE $L_{i, j}$ ) can be obtained as follows:

newEL $L_{i, j}=E L_{i, j}+$ stepsize $_{i, j}$,

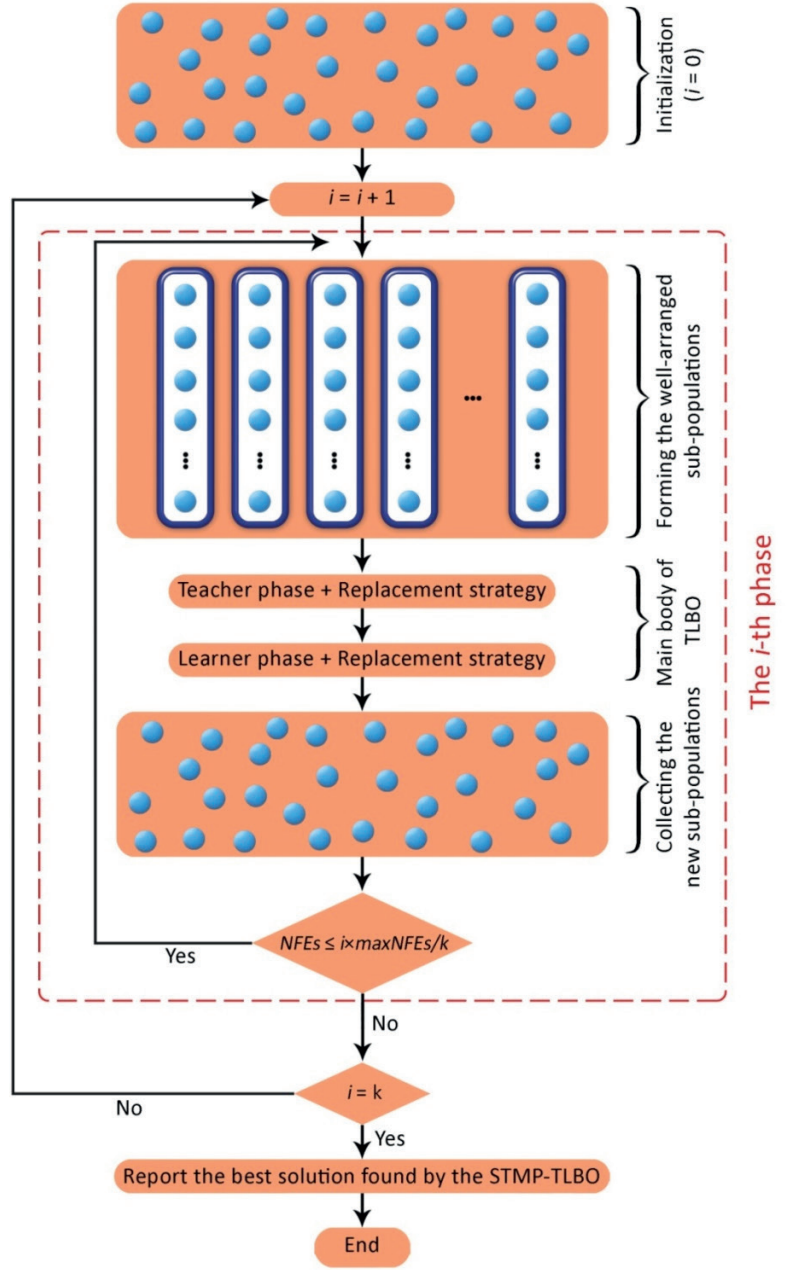

Fig. 2 Steps of the STMP-TLBO algorithm

stepsize $_{i, j}=\beta \times\left(E L_{B, j}-E L_{i, j}\right)+\alpha \times\left(E L_{i, j}-E L_{W, j}\right)$, $i=1,2, \cdots, n S$ and $j=1,2, \cdots, m$

where

$\beta=\operatorname{randi}\left(\left[\begin{array}{ll}1 & 2\end{array}\right]\right) \times \operatorname{rand}(1, n V)$,

$\alpha=$ ones $(1, n V)-\beta$.

In the above equations, $E L_{i, j}$ is the current position of the $j$-the candidate solution of the $i$-th sub-population; stepsize $_{i, j}$ is the step size of movement of the $j$-the candidate solution of the $i$-th sub-population; $E L_{B, j}$ and $E L_{W, j}$ are the better and worse candidate solutions compared to the $j$-th candidate solution of the sub-population; $n V$ is the number of design variables; $\alpha$ and $\beta$ are the parameters of the algorithm; $n S$ is the number of sub-populations; and $m$ is the number of solutions of each sub-population.

- Step four (termination criteria): If the termination condition is met, the algorithm terminates; otherwise, the algorithm returns to step two. 


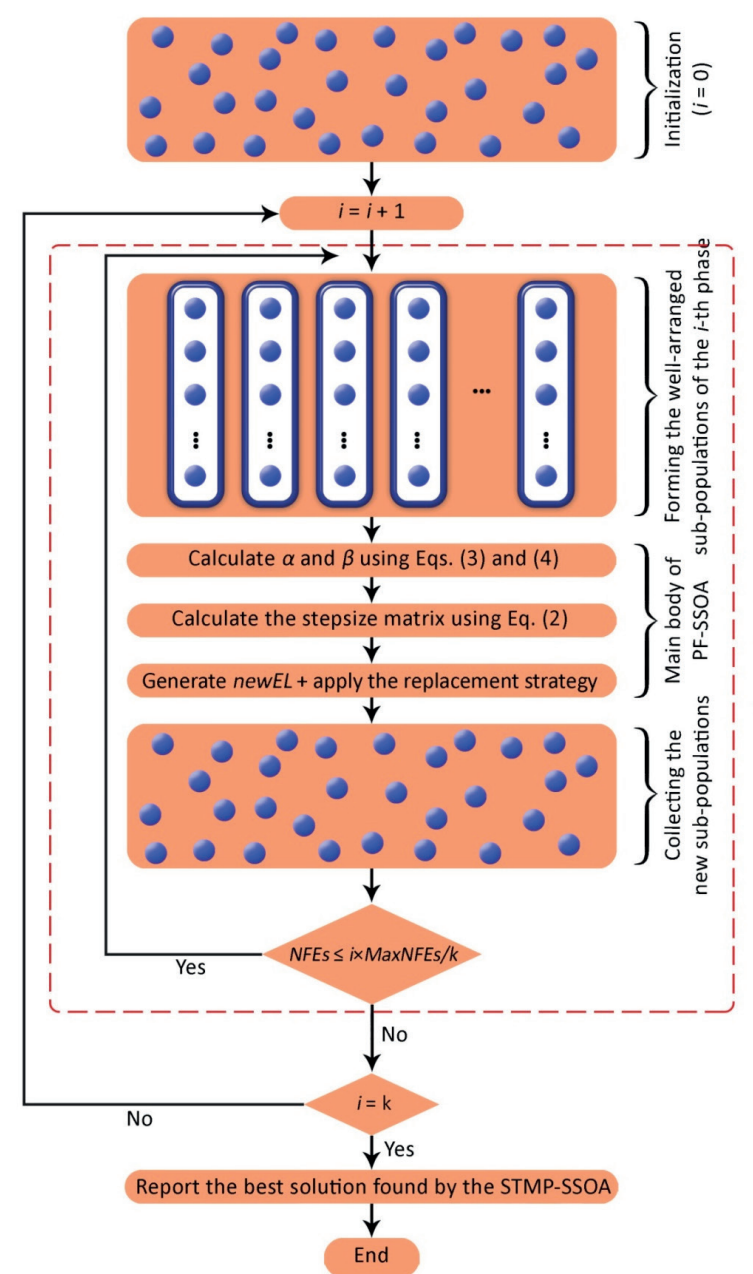

Fig. 3 Steps of the STMP-SSOA

\subsection{Set theoretical variant of the Jaya algorithm}

The Jaya algorithm is a simple population-based optimization algorithm developed by Rao [31] in 2016. The Jaya algorithm works based on a simple concept: moving toward the best candidate solution and getting away from the worst one. The candidate solutions are updated as follows:

$$
\begin{aligned}
A(i+1, j, k) & =A(i, j, k)+r(i, j, 1)(A(i, j, b)-|A(i, j, k)|) \\
& -r(i, j, 2)(A(i, j, w)-|A(i, j, k)|),
\end{aligned}
$$

where $i, j$, and $k$ are the indices of iteration number, design variable, and candidate solution, respectively; $A(i, j, k)$ means the $j$-th design variable of $k$-th candidate solution of $i$-th iteration; $r(i, j, 1)$ and $r(i, j, 2)$ are uniformly distributed random numbers in the range of $[0,1]$; and $A(i, j, b)$ and $A(i, j, w)$ are the $j$-th design variable of the best and worst candidate solutions of $i$-th iteration, respectively.

Like other P-metaheuristics, the Jaya algorithm starts with a set of randomly generated candidate solutions. Once the candidate solutions are updated, the newly generated candidate solutions are compared with the corresponding old ones in a greedy manner so that the more qualified ones are selected for the next generation. The process continues until the termination criterion is satisfied.

Based on the framework used for the OST-TLBO algorithm, the ordered set theoretical Jaya algorithm (OST-JA) is developed in five steps:

- Step one (initialization): The initial population is generated randomly within the search space.

- Step two (forming the sub-populations): This step is similar to the second step of the OST-TLBO algorithm.

- Step three (updating the candidate solutions): The candidate solutions are updated by using Eq. (5). It should be noted here that, for the purpose of updating a candidate solution, the best and worst candidate solutions must be chosen from the sub-population containing the candidate solution. Therefore, each sub-population has its own best and worst candidate solutions. This allows keeping the diversification of the search process.

- Step four (replacement strategy): The candidate solutions are updated via a simple greedy manner so that a newly generated candidate solution with better quality is preferred to the corresponding old one.

- Step five (termination criteria): If the termination criterion of the algorithm is fulfilled, the search process terminates; otherwise, the algorithm returns to the second step.

The steps of the OST-JA are shown in Fig. 4.

\section{System reliability analysis of truss structures}

In case of a statically determinate truss structure, calculation of support reactions and member forces can easily be made by the static equilibrium equations [32]. In such a truss structure, failure of any one of the structural members causes the total structure to fail. However, this is not necessarily true for redundant truss structures due to the fact that the remaining structural members may still be able to withstand the structural loading. When a member fails, the internal forces are redistributed among the remaining members. It must be mentioned that after failure of a member, its residual strength is applied to the structure and its stiffness matrix is put to zero. The residual strength of a member depends on the type of failure and the material the member made of. Afterwards, the next member to fail is determined. The process continues until the simultaneous failure of a certain number of structural members leads to the structural failure. The most common criterion of failure of a structural system is formation of a collapse mechanism, which is characterized by singularity of the structure stiffness matrix formed by the remaining members [33]. The criterion is given by: 


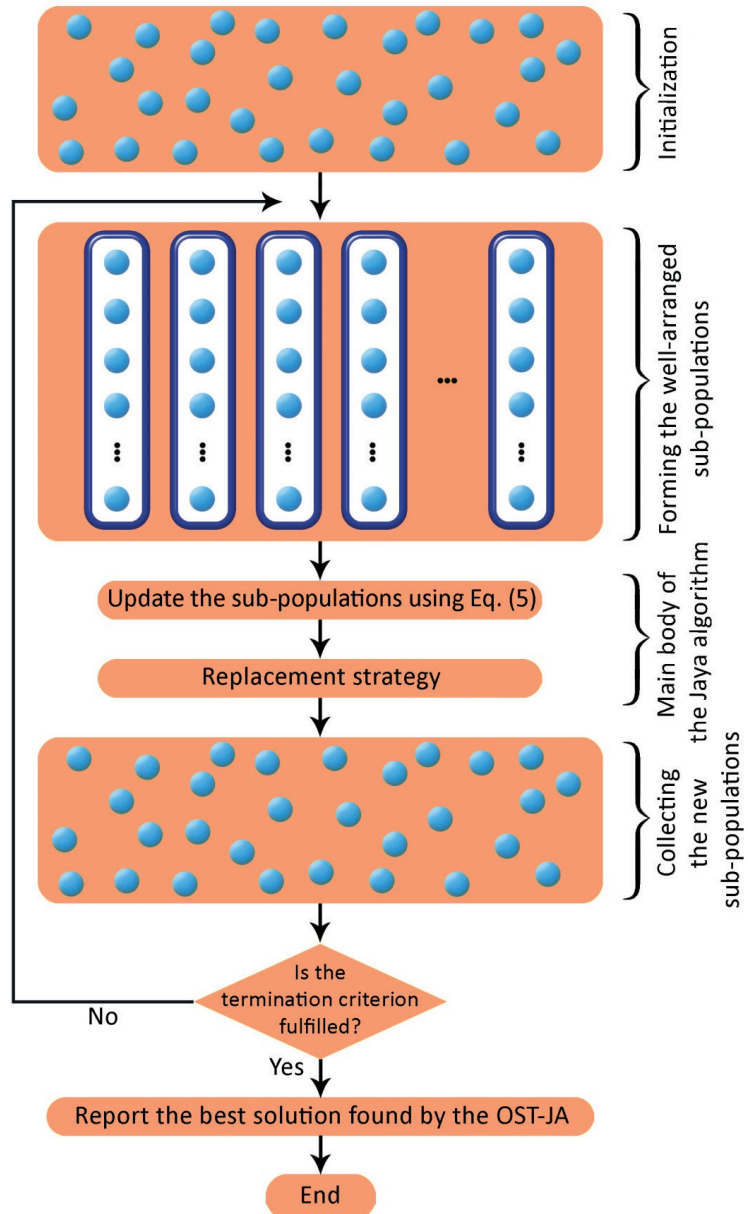

Fig. 4 Steps of the OST-JA

$\left|K^{p_{q}}\right|=0$,

where $|$.$| is the determinant of a matrix; and K^{p_{q}}$ is the structure stiffness matrix formed with the $\left(n-p_{q}\right)$ remaining members. In the following section, a systematic procedure for generation of collapse mechanisms for truss structures is expressed briefly.

\subsection{Generation of collapse mechanisms for truss structures}

Consider a space truss structure consisting of $n$ members. A member fails if the internal force exceeds the strength of the member. Therefore, the safety margin of the $i$-th member can be expressed as follows:

$$
\begin{aligned}
Z_{i} & =R_{i}\left(A_{i}, \sigma_{i}\right)-S_{i}\left(A_{1}, \cdots, A_{n} ; P_{1}, \cdots, P_{3 l} ; E_{1}, \cdots, E_{n} ; l_{1}, \cdots, l_{n}\right) \\
& =A_{i} \sigma_{i}-\left|\sum_{j=1}^{3 l} b_{i j} P_{j}\right|,
\end{aligned}
$$

where $Z_{i}, R_{i}$, and $S_{i}$ denote the safety margin, strength, and internal force of the $i$-th truss member, respectively; $A_{i}, \sigma_{i}, E_{i}$, and $l_{i}$ present the cross-sectional area, allowable stress, modulus of elasticity, and length of the $i$-th member, respectively; $n$ is the number of truss members; $P_{j}$ is the external load applied to the $j$-th degree of freedom of the structure; $l$ is the number of nodes of the structure; $|x|$ is the absolute value of $x$; and $b_{i j}$ is the internal force of the $i$-th member caused by applying a unit load to the $j$-th degree of freedom in the same direction of $P_{j}$. In case of tensile or compressive failure, the yield stress $\sigma_{y}$ is considered as the allowable stress, while the buckling stress $\sigma_{b}$ is taken when the buckling failure in compression is considered to occur. The structural failure criterion of a statically determinate truss is defined as:

$Z_{i} \leq 0 ; \forall i \in\{1,2, \cdots, n\}$.

If $R_{i}$ and $S_{i}$ are normally distributed and independent random variables, the probability of failure can be calculated as [34]:

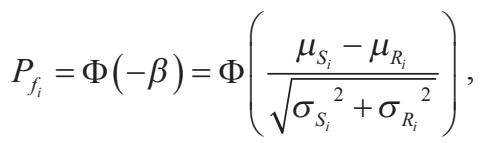

where $\mu_{S i}$ and $\sigma_{S i}$ are the mean value and standard deviation of the internal of the $i$-th truss member, respectively; $\mu_{R i}$ and $\sigma_{R i}$ are the mean value and standard deviation of the strength of the $i$-th truss member, respectively; $P_{f i}$ is the probability of failure of the $i$-th truss member; $\beta$ is the reliability index of the $i$-th truss member; and $\Phi$ is the standard normal cumulative distribution function and is calculated as:

$\Phi(X)=\int_{-\infty}^{X} \frac{1}{\sqrt{2 \pi}} \exp \left(-\frac{1}{2} x^{2}\right) d x$.

In case of a statically redundant truss structure, the structural failure criterion is defined as follows:

$Z_{m_{p}\left(m_{1}, m_{2}, \cdots, m_{p-1}\right)}^{(p)} \leq 0 ; p=1,2, \cdots, p_{q}$,

where $Z_{i\left(m_{1}, m_{2}, \cdots, m_{p-1}\right)}^{(p)}$ is given by:

$$
\begin{aligned}
& Z_{i\left(m_{1}, m_{2}, \cdots, m_{p-1}\right)}^{(p)}=A_{i} \sigma_{i} \\
& -\mid\left(\sum_{j=1}^{3 l} b_{i j}^{(p)} L_{j}-a_{i m_{1}}^{(p)} R_{m_{1}}-a_{i m_{2}}^{(p)} R_{m_{2}}-\cdots-a_{i m_{p-1}}^{(p)} R_{m_{p-1}}\right) .
\end{aligned}
$$

In the above equations, $\left(m_{1}, m_{2}, \ldots, \mathrm{m}_{p_{q}}\right)$ denotes a set of members whose simultaneous failure leads to the formation of a collapse mechanism, also known as failure mode; $\left(m_{1}, m_{2}, \ldots, \mathrm{m}_{p-1}\right)$ is the set of failed members and the order of failure; $Z_{i\left(m_{1}, m_{2}, \cdots, m_{p-1}\right)}^{(p)}$ is the safety margin of the $i$-th 
member at the $p$-th stage of failure; $b_{i j}^{(p)}$ is the internal force of the $i$-th member at the $p$-th stage of failure, which is caused by applying a unit load to the $j$-th degree of freedom in the same direction of $P_{j}$; and $a_{i j}^{(p)}$ is the internal force of the $i$-th member at the $p$-th stage of failure, which is caused by applying a unit load to both end nodes of the failed member $m_{j}$ in the opposite direction of the internal force of $m_{j}$.

A structural system fails as soon as any of its failure modes occurs. The number of possible failure modes of a real redundant structure is so astronomically large that it is impossible in practice to take into account all of them in estimating the reliability of the structure. In such a case, only the stochastically dominant failure modes are considered in the structural system reliability analysis. The dominant failure modes of structural systems can be identified using the failure path-based methods, such as the branch and bound method, the $\beta$-unzipping method, and the incremental loading method.

\subsection{The branch and bound method}

In failure path-based methods, the structural reliability analysis takes place in two main steps: (a) identification of the stochastically dominant failure modes, and (b) estimation of failure probabilities of the identified failure modes and the structural system. The branch and bound method is one of the well-known failure path-based methods developed by Murotsu et al. [35] in the late 1980s and early 1990s. The branch and bound method has three main steps as follows:

- Step one (partitioning): All the potential failure members are added to the incomplete failure path under consideration. Unlike a complete failure path, an incomplete one does not lead to the formation of a collapse mechanism. Add the newly partitioned failure paths to the set of candidate failure paths for branching. Next, calculate the upper bounds of the probabilities of failure of the partitioned failure paths by using the following upper bound:

$$
\begin{aligned}
& P_{f p\left(m_{1} m_{2} \cdots m_{p}\right)(U)} \\
& =\min _{j \in\{2, \cdots, p\}} P\left[\left(Z_{m_{1}}^{(1)} \leq 0\right) \cap\left(Z_{m_{j}\left(m_{1}, m_{2}, \cdots, m_{j-1}\right)}^{(j)} \leq 0\right)\right] .
\end{aligned}
$$

- Step two (branching): Select the failure path which gives the largest upper bound among the partitioned failure paths. Next, investigate whether a collapse mechanism is formed or not. If a collapse mechanism is not formed, go to step one and proceed the partitioning process by adding all the potential failure members to the selected failure path; otherwise, go to step three for bounding operation.
- Step three (bounding): Remove the selected failure path from the set of candidate failure paths for branching and add it to the set of the selected complete failure paths. Next, calculate the lower bound of the selected complete failure path probability by using the following equation (Eq. (14)):$$
P_{f p\left(m_{1} m_{2} \cdots m_{p}\right)(L)}
$$

$$
=\max \left[\begin{array}{l}
0, P\left[\left(Z_{m_{1}}^{(1)} \leq 0\right)\right]-P\left[\left(Z_{m_{1}}^{(1)} \leq 0\right) \cap\left(Z_{m_{2}\left(m_{1}\right)}^{(2)}>0\right)\right] \\
-\sum_{j=3}^{p} \min \left\{P_{f p\left(m_{1} m_{2} \cdots m_{p}\right)(U)}, P\left[\left(Z_{m_{1}}^{(1)} \leq 0\right) \cap\left(Z_{m_{j}\left(m_{1} m_{2} \cdots m_{j-1}\right)}^{(j)}>0\right)\right]\right\}
\end{array}\right] .
$$

Update the reference value for bounding. For this purpose, find the maximum lower bound of probability of the complete failure paths selected so far. The reference value for bounding is updated as follows:

if $R F<P_{f p\left(m_{1} m_{2} \cdots m_{p}\right)(L)} \Rightarrow R F=P_{f p\left(m_{1} m_{2} \cdots m_{p}\right)(L)}$.

Next, identify the partitioned failure paths which have the probabilities of failure lower than $R F \times 10^{-\delta}$ and remove them from the set of candidate failure paths for branching. $\delta$ is the bounding constant which is determined based on the desired accuracy in estimation of failure probabilities. In general, the larger the bounding constant, the fewer failure paths are discarded, and thus the more accurate the failure probability. If the set of candidate failure paths for branching is empty, it means that there are no further failure paths for branching. In such a case, the branch and bound method is terminated; otherwise, go to step two.

At the end of the branch and bound method, the set of the selected complete failure paths contains the stochastically dominant failure modes of the structure. Finally, the probability of failure of the structural system is estimated employing the bounding techniques and based on the dominant failure modes.

\subsection{Evaluation of the system reliability}

As mentioned earlier, a structural system will fail when the weakest failure mode occurs. Therefore, the structural system can be modelled based on a series system where the elements in the series system are failure modes. Generally, exact calculation of the probability of failure of such systems involves multi-dimensional integrals, which are complicated or even impossible to solve [36]. However, computationally simple bounding techniques exist to estimate the probability of failure of series systems, such as Cornell's bounds [37], Ditlevsen's bounds [38], and Vanmarcke's upper bound [39]. The lower and upper Cornell's bounds for the probability of failure of series systems are given by: 
$\max _{i=\{1,2, \cdots, n M\}}\left(P_{f p(U)_{i}}\right) \leq P_{f_{S}} \leq 1-\prod_{i=1}^{n M}\left(1-P_{f p(U)_{i}}\right)$

where $P_{f_{S}}$ is the probability of failure of the series system; $n M$ is the number of stochastically dominant failure modes identified by the branch and bound method; and $P_{f p(U)_{i}}$ is the upper bound of the probability of failure of the $i$-th dominant failure mode, which is determined by using Eq. (11).

\section{System reliability-based design optimization of truss structures}

The main goal of structural reliability theory is to make it possible to design structures based on the reliability concepts. It has been recognized that the actual load-carrying capacity of a structural system is usually much higher than what is obtained by the design of individual structural members, which may be attributed to the interaction between the structural members forming the structural system [40]. Therefore, the SRBDO approaches should be used. The SRBDO approaches mainly aim to minimize the structural weight under the constraint on the probability of failure of the structural system. The problem of system reliability-based design optimization of truss structures can be expressed mathematically as follows:

Find

$$
\{\boldsymbol{X}\}=\left[x_{1}, x_{2}, \cdots, x_{n D V}\right] ; x_{i} \in D_{i},
$$

to minimize $W \quad(\{\boldsymbol{X}\})=\sum_{i=1}^{n} \rho_{i} A_{i} l_{i}$,

so that:

1. The truss structure would be geometrically stable;

2. The following constraints would not be violated.

$\left\{\begin{array}{c}x_{i}^{L} \leq x_{i} \leq x_{i}^{U} ; i=1,2, \cdots, n D V \\ P_{f}(\{\boldsymbol{X}\}) \leq P_{f}^{t}=\Phi\left(-\beta_{t}\right)\end{array}\right.$

In the above equations, $\{\boldsymbol{X}\}$ is the candidate solution vector containing the design variables; $x_{i}$ denotes the $i$-th design variable; $n D V$ is the number of design variables; $D_{i}$ is the allowable set of continuous values for the design variable $x_{i} ; W(\{\boldsymbol{X}\})$ presents the total weight of the structure; $p_{i}$ is the material density of the $i$-th truss member, respectively; $x_{i}^{L}$ and $x_{i}^{U}$ are the lower and upper bounds of the design variable $x_{i}$, respectively; $P_{f}\{\boldsymbol{X}\}$ denotes the upper bound of the probability of failure of the structure, which is obtained by the branch and bound method; $\beta_{t}$ is the target system reliability index; and $P_{f}^{t}$ is the target failure probability of the structure. The design variable $x_{i}$ can vary continuously within a certain range as follows:

$D_{i}=\left\{x_{i} \mid x_{i} \in\left[x_{i}^{L}, x_{i}^{U}\right]\right\}$.

\section{Case study}

To investigate the performance of the proposed SRBDO approach, three truss optimization problems with constraints on the target failure probability are considered as follows: size optimization of a statically indeterminate 16-member planar truss; size optimization of a statically indeterminate 65-member truss bridge; and size optimization of a statically indeterminate 67-member truss bridge. It should be noted that since there are no direct comparative SRBDO studies available in the literature for some of these truss structures, the results are compared with deterministic solutions, if possible. The aim of optimization is to minimize the structural weight while satisfying constraint on the structural probability of failure. The cross-sectional areas of the members and the coordinates of the nodes are considered as continuous design variables. The members are assumed to be circular solid sections made of a perfectly ductile material. The loads and the strengths of structural members are considered to be normally distributed uncorrelated random variables. Buckling failure mode in compression members is also considered. In the case of including buckling failure mode, the mean and coefficient of variation of buckling stress of the member with initial deflection under compression are evaluated by the following formulas [35]:

$\mu_{\sigma_{b}}=\frac{1}{2}\left(\mu_{\sigma_{y}}+\sigma_{E}+\frac{\mu_{W_{0}}}{S} \sigma_{E}\right)\left(1-\sqrt{1-\left(4 \mu_{\sigma_{y}} \sigma_{E}\right) /\left(\mu_{\sigma_{y}}+\sigma_{E}+\frac{\mu_{W_{0}}}{S} \sigma_{E}\right)^{2}}\right)$

$C V_{\sigma_{b}}=\frac{\sqrt{\left(C V_{\sigma_{y}} \mu_{\sigma_{y}}\right)^{2}+\left(\sigma_{E} / S\right)^{2}\left(C V_{W_{0}} \mu_{W_{0}}\right)^{2}}}{\left(\mu_{\sigma_{y}}+\sigma_{E}+\frac{\mu_{W_{0}}}{S} \sigma_{E}\right) \sqrt{1-\left(4 \mu_{\sigma_{y}} \sigma_{E}\right) /\left(\mu_{\sigma_{y}}+\sigma_{E}+\frac{\mu_{W_{0}}}{S} \sigma_{E}\right)^{2}}}$

where $\mu_{\sigma_{v}}$ and $C V_{\sigma_{v}}$ are the mean and coefficient of variation of the yield stress; $\mu_{W_{0}}$ and $C V_{W_{0}}$ are the mean and coefficient of variation of the initial deflection of the members; $S$ is the radius of gyration of the cross-sectional area of the members; and $\sigma_{E}=\pi^{2} E /(l / S)^{2}$ is the Euler buckling stress. In the above equation, initial deflection and yield stress are treated as normally distributed uncorrelated random variables. The termination criterion of the algorithms is considered to be the maximum number of objective function evaluations (MaxNFEs). The optimization algorithms, as well as structural reliability analysis codes, are implemented in Matlab. 


\subsection{Statically indeterminate 16-member planar truss}

The first example is size optimization of a 16-member truss with three degrees of redundancy shown in Fig. 5. Reliability analysis of this structure was previously done by many researchers such as Murotsu et al. [35], Biabani Hamedani and Kalatjari [27], and so on. The layout of the structure is kept unchanged during the optimization process. Truss members are divided into eight groups of design variables. The design optimization is carried out for both the cases of with and without considering the effect of buckling failure in compression members. Table 1 lists the numerical data of the structure, such as design variable bounds, material properties, statistical data of random variables, etc. The optimum design solutions for the case of neglecting the effect of buckling failure are listed in Table 2 for various set theoretical optimization algorithms. Table 3 presents the optimum design solutions for the case where buckling failure is included. As Tables 2 and 3 prove, constraint on the probability of failure is satisfied in all cases. The results show that, in the case where buckling failure is not considered, the best designs of OST-TLBO, STMP-TLBO, STMP-SSOA, and OST-JA are very close to each other, while, in the case where buckling failure is considered, STMP-SSOA and OST-JA perform better than OST-TLBO and STMP-TLBO in terms of the best weight. A comparison between the two cases results (with and without considering buckling failure) indicates that considering buckling failure mode in compression members significantly affects the optimal values of design variables. Consequently, buckling failure mode should be taken into account in the optimum design of truss

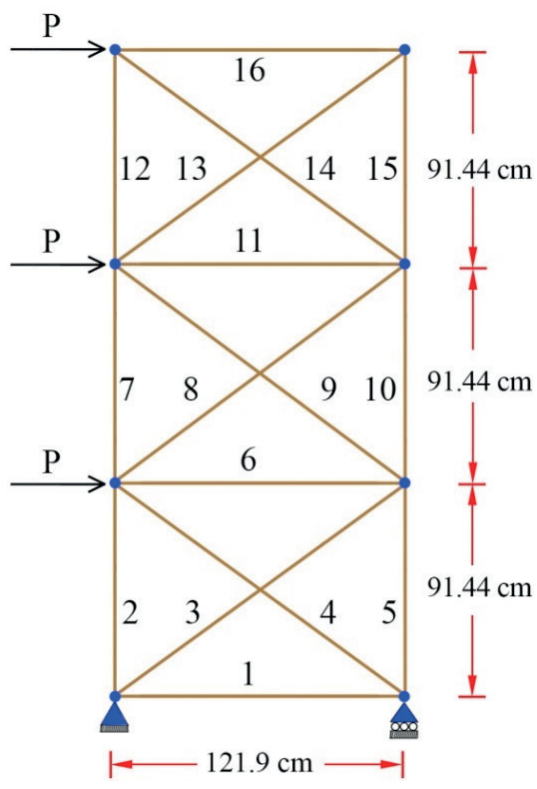

Fig. 5 Statically indeterminate 16-member truss structures. The convergence histories of the best optimum designs are presented in Figs. 6 and 7. It can be observed from both Figs. 6 and 7 that STMP-SSOA and OST-JA converge faster than OST-TLBO and STMP-TLBO.

Table 1 Data of statically indeterminate 16-member truss

\begin{tabular}{lc}
\hline Property / Unit & Value \\
\hline$E$ (Modulus of elasticity) / $G P a$ & 206 \\
$\rho$ (Material density) / $\mathrm{kg} / \mathrm{m}^{3}$ & 2700 \\
$\mu_{\sigma_{y}}$ (Mean value of yield stress) / MPa & 276 \\
$C V_{\sigma_{y}}$ (Coefficient of variation of yield stress) & 0.02 \\
$\mu_{P}$ (Mean value of applied loads) / $k N$ & 44.45 \\
$C V_{P}$ (Coefficient of variation of applied loads) & 0.02 \\
$\quad \mu_{W_{0}}$ & 0.1 \\
$C V_{W_{0}}$ (Coefficient of variation of initial deflection) & 0.1 \\
Lower bound of design variables / $\mathrm{cm}^{2}$ & 0.1 \\
Upper bound of design variables / $\mathrm{cm}^{2}$ & 15 \\
$P_{f}^{t}$ (Target failure probability) & $10^{-5}$ \\
\hline
\end{tabular}

Table 2 Comparison of optimization results for the 16-member truss (buckling failure is neglected)

\begin{tabular}{lccccc}
\hline $\begin{array}{l}\text { Element } \\
\text { groups }\end{array}$ & $A_{i}\left(\mathrm{~cm}_{2}\right)$ & $\begin{array}{c}\text { OST- } \\
\text { TLBO }\end{array}$ & $\begin{array}{c}\text { STMP- } \\
\text { TLBO }\end{array}$ & $\begin{array}{c}\text { STMP- } \\
\text { SSOA }\end{array}$ & OST-JA \\
\hline 1 & $A_{1}$ & 3.59 & 3.60 & 3.62 & 3.62 \\
2,5 & $A_{2}$ & 7.58 & 7.56 & 7.58 & 7.58 \\
$3,4,14$ & $A_{3}$ & 3.26 & 3.26 & 3.26 & 3.26 \\
6 & $A_{4}$ & 1.54 & 1.51 & 1.54 & 1.55 \\
$7,8,10$ & $A_{5}$ & 3.47 & 3.48 & 3.47 & 3.47 \\
9 & $A_{6}$ & 2.59 & 2.63 & 2.59 & 2.59 \\
$11,12,15$ & $A_{7}$ & 1.81 & 1.80 & 1.81 & 1.81 \\
13,16 & $A_{8}$ & 0.10 & 0.11 & 0.10 & 0.10 \\
$P_{f}$ & & $10^{-5}$ & $9.96 \times 10^{-6}$ & $9.96 \times 10^{-6}$ & $9.92 \times 10^{-6}$ \\
Best Weight $(\mathrm{kg})$ & 15.23 & 15.24 & 15.23 & 15.23 \\
No. of analyses & 5000 & 5000 & 5000 & 5000 \\
\hline
\end{tabular}

Table 3 Comparison of optimization results for the 16-member truss (buckling failure is included)

\begin{tabular}{lccccc}
\hline $\begin{array}{l}\text { Element } \\
\text { groups }\end{array}$ & $A_{i}\left(\mathrm{~cm}_{2}\right)$ & $\begin{array}{c}\text { OST- } \\
\text { TLBO }\end{array}$ & $\begin{array}{c}\text { STMP- } \\
\text { TLBO }\end{array}$ & $\begin{array}{c}\text { STMP- } \\
\text { SSOA }\end{array}$ & OST-JA \\
\hline 1 & $A_{1}$ & 2.41 & 3.20 & 3.13 & 3.13 \\
2,5 & $A_{2}$ & 12.66 & 12.11 & 12.09 & 12.09 \\
$3,4,14$ & $A_{3}$ & 11.26 & 11.24 & 11.28 & 11.29 \\
6 & $A_{4}$ & 5.96 & 0.15 & 0.10 & 0.10 \\
$7,8,10$ & $A_{5}$ & 7.16 & 6.70 & 6.62 & 6.61 \\
9 & $A_{6}$ & 11.33 & 14.36 & 14.28 & 14.25 \\
$11,12,15$ & $A_{7}$ & 1.87 & 1.96 & 1.94 & 1.94 \\
13,16 & $A_{8}$ & 0.11 & 0.17 & 0.10 & 0.10 \\
$P_{f}$ & & $9.94 \times 10^{-6}$ & $9.94 \times 10^{-6}$ & $9.94 \times 10^{-6}$ & $9.99 \times 10^{-6}$ \\
Best Weight $(\mathrm{kg})$ & 35.67 & 34.66 & 34.49 & 34.49 \\
No. of analyses & 5000 & 5000 & 5000 & 5000 \\
\hline
\end{tabular}




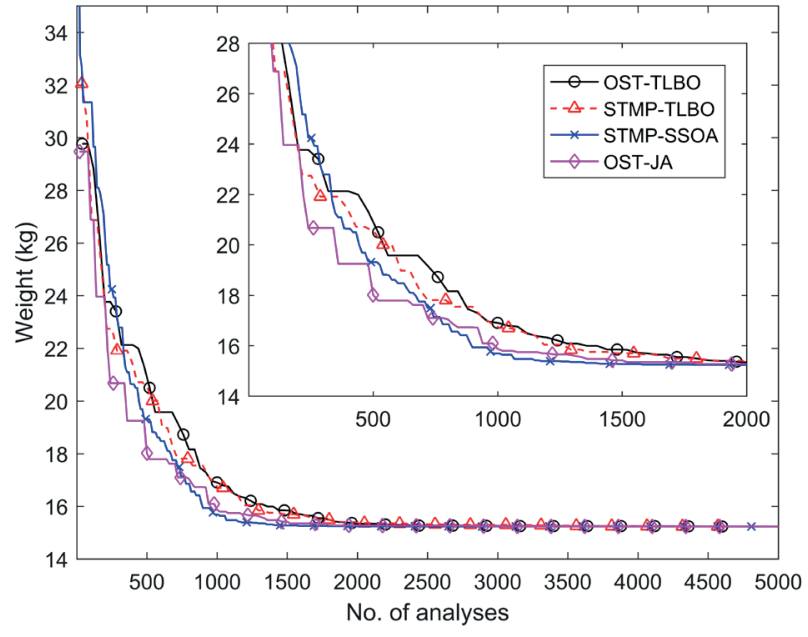

Fig. 6 Convergence histories of the statically redundant 16-member truss (buckling failure is neglected)

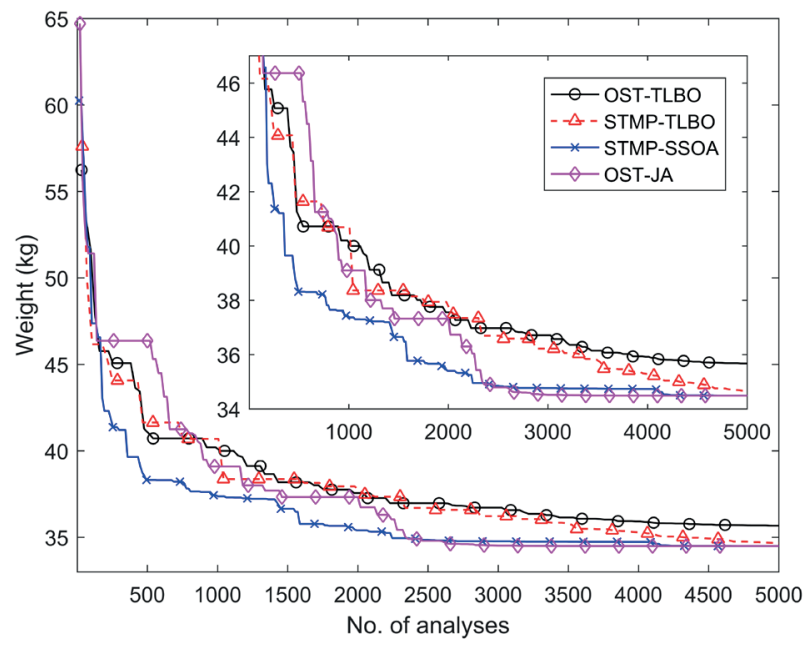

Fig. 7 Convergence histories of the statically redundant 16-member truss (buckling failure is included)

\subsection{Statically indeterminate 65 -member truss bridge}

The next example is size optimization of a 65 -member truss bridge with eight design variables as presented in Fig. 8. The layout and geometry of the structure are taken from Nakib [14]. The structure has only one degree of redundancy. The structural members are assumed to fail only in tension or compression. Employing the symmetry of the structure, the structural members are categorized into eight different groups of design variables. Table 4 summarizes the material properties, design variables bounds, and statistical data of random variables of the truss bridge. The target failure probability of the structure is equal to $10^{-5}$. This example was previously studied by Nakib [14] in terms of deterministic optimization. Table 5 compares the optimum designs of the 65 -member truss bridge obtained by various set theoretical optimization algorithms. As can be

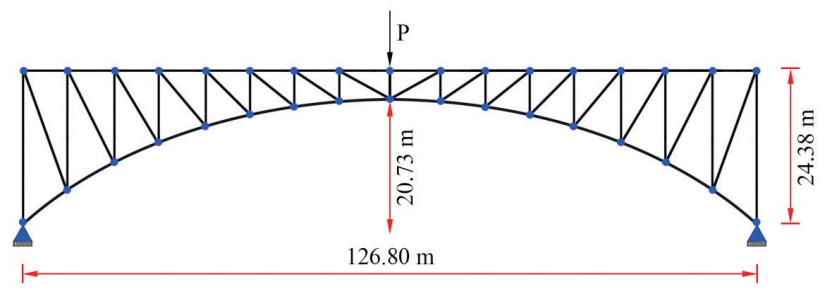

Fig. 8 Statically indeterminate 65-member truss bridge

Table 4 Data of statically indeterminate 65 -member truss bridge

\begin{tabular}{lc}
\hline Property / Unit & Value \\
\hline$E$ (Modulus of elasticity) / GPa & 206 \\
$\rho$ (Material density) $/ \mathrm{kg} / \mathrm{m}^{3}$ & 2700 \\
$\mu_{\sigma_{y}}$ (Mean value of yield stress) / MPa & 276 \\
$C V_{\sigma_{y}}$ (Coefficient of variation of yield stress) & 0.02 \\
$\mu_{P}$ (Mean value of applied loads) / $\mathrm{kN}$ & 44.45 \\
$C V_{P}$ (Coefficient of variation of applied loads) & 0.02 \\
Lower bound of design variables / $\mathrm{cm}^{2}$ & 0.1 \\
Upper bound of design variables / $\mathrm{cm}^{2}$ & 15 \\
$P_{f}^{t}$ (Target failure probability) & $10^{-5}$ \\
\hline
\end{tabular}

seen from the table, the optimum designs of the 65 -member truss bridge are very close to each other in terms of structural weight and cross-sectional areas of members. Fig. 9 compares the convergence histories of the best optimum designs of 65 -member truss bridge. It is found that OST-JA converges significantly faster than the other set theoretical metaheuristics in this problem.

\subsection{Statically indeterminate 67 -member truss bridge}

The last example investigates the effect of ductile redundant members added to the 65 -member truss bridge. Fig. 10 shows a 67 -member statically indeterminate truss bridge which is formed by adding two redundant members to the 65 -member truss bridge. In such a case, the resulting structure has three degrees of redundancy. Therefore, this is a size optimization problem with nine groups of design variables (see Table 6). The numerical data are the same as those of the previous example (see Table 4). Table 6 presents the SRBDO results of the 67-member statically indeterminate truss bridge. The optimum designs found by OST-TLBO, STMP-TLBO, STMP-SSOA, and OST-JA have the weights of $2368.29 \mathrm{~kg}, 2373.39 \mathrm{~kg}, 2378.60 \mathrm{~kg}$, and $2368.03 \mathrm{~kg}$, respectively, which are $15.27 \%, 15.09 \%$, $14.90 \%$, and $15.27 \%$ less than those of the $65-\mathrm{mem}-$ ber truss bridge, respectively. The results prove that the addition of ductile redundant members to the 65 -member truss bridge significantly decreases the optimized structural weight. This is due to the fact that the load carrying 
Table 5 Comparison of optimization results for the statically indeterminate 65 -member truss bridge

\begin{tabular}{lcccccc}
\hline \multirow{2}{*}{ Element groups } & $A_{i}\left(\mathrm{~cm}_{2}\right)$ & \multicolumn{3}{c}{ Reliability-based approach } & \multicolumn{3}{c}{ Deterministic approach } \\
\hline $2,6,10,14,50,54,58,62$ & $A_{1}$ & 8.35 & 8.35 & 8.34 & 8.35 & 6.53 \\
$18,22,26,30,34,38,42,46$ & $A_{2}$ & 7.24 & 7.26 & 7.24 & 7.24 & 5.57 \\
$4,8,12,16,52,56,60,64$ & $A_{3}$ & 0.65 & 0.65 & 0.65 & 0.65 & 0.65 \\
$20,24,28,32,36,40,44,48$ & $A_{4}$ & 19.07 & 19.04 & 19.09 & 19.07 & 16.21 \\
$1,5,9,13,53,57,61,65$ & $A_{5}$ & 0.80 & 0.80 & 0.80 & 0.80 & 0.88 \\
$17,21,25,29,33,37,41,45,49$ & $A_{6}$ & 6.80 & 6.80 & 6.81 & 6.80 & 5.37 \\
$3,7,11,15,51,55,59,63$ & $A_{7}$ & 0.89 & 0.89 & 0.89 & 0.89 & 0.95 \\
$19,23,27,31,35,39,43,47$ & $A_{8}$ & 8.71 & 8.72 & 8.71 & 8.71 & 6.83 \\
$P_{f}$ & & $100^{-5}$ & $10^{-5}$ & $10^{-5}$ & $10^{-5}$ & - \\
Best Weight $(\mathrm{kg})$ & & 2794.94 & 2795.23 & 2794.98 & 2794.87 & 6000 \\
No. of analyses & & 6000 & 6000 & 6000 & 6000 & 2286.16 \\
\hline
\end{tabular}

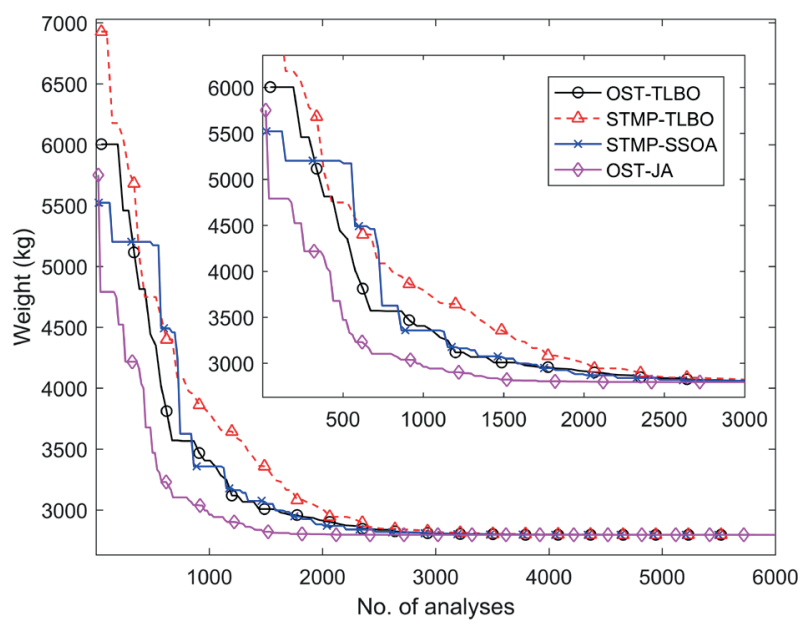

Fig. 9 Convergence histories of the statically redundant 65 -member truss bridge

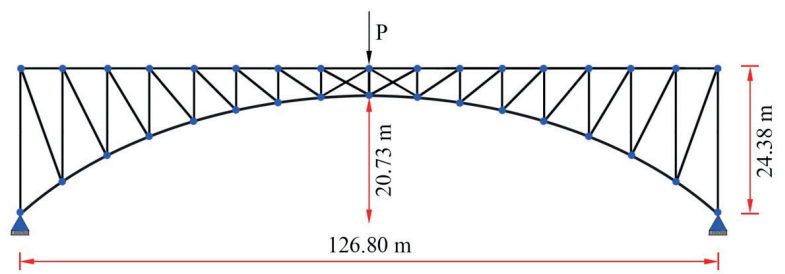

Fig. 10 Statically indeterminate 67-member truss bridge

capacity of a structure that is designed with a high degree of redundancy is often much larger than that of a structure with lower degree of redundancy. Fig. 11 shows convergence histories of the best designs of 67-member truss bridge. As can be seen from the figure, OST-JA has better convergence performance compared to the other set theoretical optimization algorithms in this problem.

Table 6 Comparison of optimization results for the statically indeterminate 65 -member truss bridge

\begin{tabular}{|c|c|c|c|c|c|}
\hline \multirow{2}{*}{ Element groups } & \multirow{2}{*}{$A_{i}\left(\mathrm{~cm}_{2}\right)$} & \multicolumn{4}{|c|}{ Reliability-based approach } \\
\hline & & OST-TLBO & STMP-TLBO & STMP-SSOA & OST-JA \\
\hline $2,6,10,14,50,54,58,62$ & $A_{1}$ & 9.61 & 9.48 & 9.63 & 9.60 \\
\hline $18,22,26,30,34,38,42,46$ & $A_{2}$ & 9.11 & 8.93 & 9.08 & 9.13 \\
\hline $4,8,12,16,52,56,60,64$ & $A_{3}$ & 1.07 & 0.99 & 1.05 & 1.07 \\
\hline $20,24,28,32,36,40,44,48$ & $A_{4}$ & 9.17 & 9.82 & 9.16 & 9.16 \\
\hline $1,5,9,13,53,57,61,65$ & $A_{5}$ & 1.12 & 1.07 & 1.11 & 1.12 \\
\hline $17,21,25,29,33,37,41,45,49$ & $A_{6}$ & 2.91 & 2.97 & 2.99 & 2.91 \\
\hline $3,7,11,15,51,55,59,63$ & $A_{7}$ & 1.27 & 1.22 & 1.30 & 1.27 \\
\hline $19,23,27,31,35,39,43,47$ & $A_{8}$ & 6.32 & 6.44 & 6.37 & 6.32 \\
\hline 66,67 & $A_{9}$ & 7.96 & 7.61 & 8.28 & 7.97 \\
\hline$P_{f}$ & & $9.96 \times 10^{-6}$ & $9.85 \times 10^{-6}$ & $9.68 \times 10^{-6}$ & $10^{-5}$ \\
\hline Best Weight (kg) & & 2368.29 & 2373.39 & 2378.60 & 2368.03 \\
\hline No. of analyses & & 6000 & 6000 & 6000 & 6000 \\
\hline
\end{tabular}




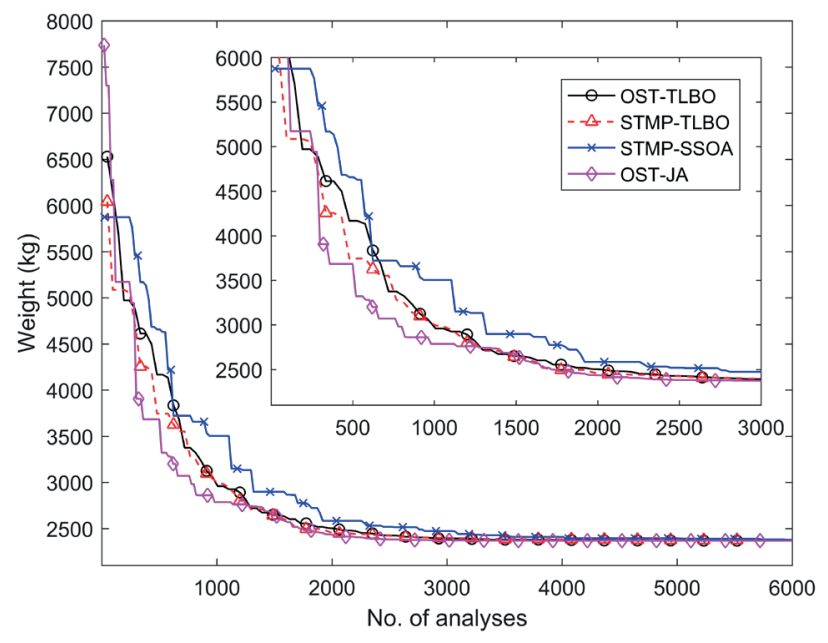

Fig. 11 Convergence histories of the statically redundant 67-member truss bridge

\section{Conclusions}

This paper presents an approach to solve the SRBDO problem of truss structures by employing a failure pathbased method, called the branch and bound method, for structural system reliability analysis and a general set theoretical framework proposed by Kaveh et al. [6] for

\section{References}

[1] Tejani, G., Savsani, V., Bureerat, S. "Truss Topology Optimization: A Review", Scholars' Press, Beau Bassin, Mauritius, 2018. [online] Available at: https://www.researchgate.net/ publication/326377131_Truss_Topology_Optimization_A_Review

[2] Dhingra, A. K., Bennage, W. A. "Topological optimization of truss structures using simulated annealing", Engineering Optimization, 24(4), pp. 239-259, 1995.

https://doi.org/10.1080/03052159508941192

[3] Kaveh, A., Khayatazad, M. "Ray optimization for size and shape optimization of truss structures", Computers \& Structures, 117, pp. 82-94, 2013.

https://doi.org/10.1016/j.compstruc.2012.12.010

[4] Hasançebi, O., Kazemzadeh Azad, S. "Discrete size optimization of steel trusses using a refined big bang-big crunch algorithm", Engineering Optimization, 46(1), pp. 61-83, 2014.

https://doi.org/10.1080/0305215X.2012.748047

[5] Hasançebi, O., Kazemzadeh Azad, S. "Adaptive dimensional search: A new metaheuristic algorithm for discrete truss sizing optimization", Computers \& Structures, 154, pp. 1-16, 2015.

https://doi.org/10.1016/j.compstruc.2015.03.014

[6] Kaveh, A., Biabani Hamedani, K., Kamalinejad, M. "Set theoretical variants of the teaching-learning-based optimization algorithm for optimal design of truss structures with multiple frequency constraints", Acta Mechanica, 231, pp. 3645-3672, 2020.

https://doi.org/10.1007/s00707-020-02718-3

[7] Kaveh, A., Kamalinejad, M., Biabani Hamedani, K. "Enhanced versions of the shuffled shepherd optimization algorithm for optimal design of skeletal structures", Structures, 29, pp. 1463-1495, 2021. https://doi.org/10.1016/j.istruc.2020.12.032 population-based metaheuristics. The main idea of the framework is to divide the population of candidate solutions into a number of smaller well-arranged sub-populations, with the aim of improving the compromise between exploration and exploitation of the search. Furthermore, searching via several sub-populations allows keeping the diversity of the search and thus improves the ability to escape from local optima. The set theoretical variants of three P-metaheuristics, including TLBO, SSOA, and Jaya algorithm, are employed for solving the considered problems. The robustness of the approach is validated through three truss structure examples. The results show the effectiveness and applicability of the set theoretical optimization algorithms for the optimum design of truss structures subject to system reliability constraints. A major advantage of the proposed SRBDO approach is that, due to the use of a systems approach for calculation of structural reliability, a more accurate estimation of the load-carrying capacity of truss structures is made, and thus more realistic optimization results can be obtained. This would effectively help to strike a suitable balance between minimum weight and target safety.

[8] Lógó, J., Movahedi Rad, M., Hjiaj, M. "Plastic Behaviour and Stability Constraints in the Reliability based Shakedown Analysis and Optimal Design of Skeletal Structures", In: Topping, B. H. V., Adam, J. M., Pallarés, F. J., Bru, R., Romero, M. L. (eds.) Proceedings of the Tenth International Conference on Computational Structures Technology, Civil-Comp Press, Stirlingshire, UK, Paper 203, 2010. https://doi.org/10.4203/ccp.93.203

[9] Lógó, J., Movahedi Rad, M., Knabel, J., Tauzowski, P. "Reliability based design of frames with limited residual strain energy capacity", Periodica Polytechnica Civil Engineering, 55(1), pp. 13-20, 2011. https://doi.org/10.3311/pp.ci.2011-1.02

[10] Movahedi Rad, M. "Reliability Based Analysis and Optimum Design of Laterally Loaded Piles", Periodica Polytechnica Civil Engineering, 61(3), pp. 491-497, 2017. https://doi.org/10.3311/PPci.8756

[11] Nakib, R., Frangopol, D. M. "RBSA and RBSA-OPT: Two computer programs for structural system reliability analysis and optimization", Computers \& Structures, 36(1), pp. 13-27, 1990. https://doi.org/10.1016/0045-7949(90)90170-7

[12] Murotsu, Y., Shao, S. "Optimum shape design of truss structures based on reliability", Structural Optimization, 2, pp. 65-76, 1990. https://doi.org/10.1007/BF01745455

[13] Murotsu, Y., Shao, S., Watanabe, A. "An approach to reliability-based optimization of redundant structures", Structural Safety, 16(1-2), pp. 133-143, 1994. https://doi.org/10.1016/0167-4730(94)00031-K

[14] Nakib, R. "Deterministic and reliability-based optimization of truss bridges", Computers \& Structures, 65(5), pp. 767-775, 1997. https://doi.org/10.1016/S0045-7949(94)E0289-E 
[15] Stocki, R., Kolanek, K., Jendo, S., Kleiber, M. "Study on discrete optimization techniques in reliability-based optimization of truss structures", Computers \& Structures, 79, pp. 2235-2247, 2001. https://doi.org/10.1016/S0045-7949(01)00080-3

[16] Burton, S. A., Hajela, P. "Reliability-based shape optimization of truss structures", presented at 19th AIAA Applied Aerodynamics Conference, Anaheim, CA, USA, June, 11-14, 2001. https://doi.org/10.2514/6.2001-1681

[17] Dimou, C. K., Koumousis, V. K. "Reliability-based optimal design of truss structures using particle swarm optimization", Journal of Computing in Civil Engineering, 23(2), pp. 100-109, 2009. https://doi.org/10.1061/(ASCE)0887-3801(2009)23:2(100)

[18] Liu, Y., Lu, N., Noori, M., Yin, X. "System reliability-based optimisation for truss structures using genetic algorithm and neural network", International Journal of Reliability and Safety (IJRS), 8(1), pp. 51-69, 2014.

https://doi.org/10.1504/IJRS.2014.062640

[19] Lopez, R. H., Torii, A. J., Miguel, L. F. F., De Cursi, J. E. S. "An approach for the global reliability based optimization of the size and shape of truss structures", Mechanics \& Industry, 16(6), Article No. 603, 2015.

https://doi.org/10.1051/meca/2015029

[20] Cheng, J., Jin, H. "Reliability-based optimization of steel truss arch bridges", International Journal of Steel Structures, 17(4), pp. 1415$1425,2017$. https://doi.org/10.1007/s13296-017-1212-y

[21] Truong, V. H., Kim, S. E. "Reliability-based design optimization of nonlinear inelastic trusses using improved differential evolution algorithm", Advances in Engineering Software, 121, pp. 59-74, 2018. https://doi.org/10.1016/j.advengsoft.2018.03.006

[22] Palizzolo, L., Tabbuso, P. "Reliability-based design optimization of trusses under dynamic shakedown constraints", Structural and Multidisciplinary Optimization, 60, pp. 1097-1108, 2019. https://doi.org/10.1007/s00158-019-02259-x

[23] Zaeimi, M., Ghoddosian, A. "System reliability based design optimization of truss structures with interval variables", Periodica Polytechnica Civil Engineering, 64(1), pp. 42-59, 2020. https://doi.org/10.3311/PPci.14238

[24] Csébfalvi, A., Lógó, J. "A critical analysis of expected-compliance model in volume-constrained robust topology optimization with normally distributed loading directions, using a minimax-compliance approach alternatively", Advances in Engineering Software, 120, pp. 107-115, 2018. https://doi.org/10.1016/j.advengsoft.2018.02.003

[25] Csébfalvi, A. "Critical Investigation of the Combined Compliance Average and Spreading Measures in the Robust Topology Optimization with Uncertain Loading Magnitude and Direction", Periodica Polytechnica Civil Engineering, 64(4), pp. 1275-1283, 2020. https://doi.org/10.3311/PPci.16681

[26] Csébfalvi, A., Lógó, J. "Investigation of the possible numerical treatments of a compliance-function-shape-oriented robust truss sizing model with uncertain loading directions", Advances in Engineering Software, 149, Article No. 102899, 2020. https://doi.org/10.1016/j.advengsoft.2020.102899
[27] Biabani Hamedani, K., Kalatjari, V. R. "Structural system reliability-based optimization of truss structures using genetic algorithm", International Journal of Optimization in Civil Engineering, 8(4), pp. 565-586, 2018. [online] Available at: http://ijoce.iust.ac.ir/article-1363-en.html

[28] Park, S., Choi, S., Sikorsky, C., Stubbs, N. "Efficient method for calculation of system reliability of a complex structure", International Journal of Solids and Structures, 41(18-19), pp. 5035-5050, 2004. https://doi.org/10.1016/j.ijsolstr.2004.04.028

[29] Kaveh, A., Biabani Hamedani, K., Zaerreza, A. "A set theoretical shuffled shepherd optimization algorithm for optimal design of cantilever retaining wall structures", Engineering with Computers, 2020. https://doi.org/10.1007/s00366-020-00999-9

[30] Rao, R. V., Savsani, V. J., Vakharia, D. P. "Teaching-learningbased optimization: A novel method for constrained mechanical design optimization problems", Computer-Aided Design, 43, pp. 303-315, 2011.

https://doi.org/10.1016/j.cad.2010.12.015

[31] Rao, R. V. "Jaya: A simple and new optimization algorithm for solving constrained and unconstrained optimization problems", International Journal of Industrial Engineering Computations, 7, pp. 19-34, 2016.

https://doi.org/10.5267/j.ijiec.2015.8.004

[32] Wang, H., Yu, W., Chen, G. "An approach of topology optimization of multi-rigid-body mechanism", Computer-Aided Design, 84, pp. 39-55, 2017.

https://doi.org/10.1016/j.cad.2016.12.002

[33] Tang, L. K., Melchers, R. E. "Dominant mechanisms in stochastic plastic frames", Reliability Engineering, 18(2), pp. 101-115, 1987. https://doi.org/10.1016/0143-8174(87)90025-4

[34] Zaeimi, M., Ghoddosian, A. "Structural reliability assessment based on the improved constrained differential evolution algorithm", Periodica Polytechnica Civil Engineering, 62(2), pp. 494-507, 2018. https://doi.org/10.3311/PPci.11537

[35] Murotsu, Y., Okada, H., Niwa, K., Miwa, S. "New Method for Evaluating Lower and Upper Bounds of Failure Probability in Redundant Truss Structures", Bulletin of University of Osaka Prefecture, Series A, Engineering and Natural Sciences, 28(1), pp. 79-91, 1979. http://doi.org/10.24729/00008657

[36] Kaveh, A., Javadi, S. M., Mahdipour Moghanni, R. "Reliability Analysis via an Optimal Covariance Matrix Adaptation Evolution Strategy: Emphasis on Applications in Civil Engineering", Periodica Polytechnica Civil Engineering, 64(2), pp. 579-588, 2020. https://doi.org/10.3311/PPci.15793

[37] Cornell, C. A. "Bounds on the Reliability of Structural Systems", Journal of the Structural Division, 93(1), pp. 171-200, 1967.

[38] Ditlevsen, O. "Narrow Reliability Bounds for Structural Systems", Journal of Structural Mechanics, 7(4), pp. 453-472, 1979. https://doi.org/10.1080/03601217908905329

[39] Vanmarcke, E. H. "Matrix formulation of reliability analysis and reliability-based design", Computers \& Structures, 3(4), pp. 757$770,1973$.

https://doi.org/10.1016/0045-7949(73)90056-4

[40] Nowak, A. S. "System reliability models for bridge structures", Bulletin of the Polish Academy of Sciences, 52(4), pp. 321-328, 2004. 